\title{
Wirtschaftlichkeit gegen Qualität
}

\author{
Am gleichen Tag im Januar hat santésuisse beachtenswerte Positionspapiere \\ zu den Themen Qualitätssicherung und Wirtschaftlichkeitsverfahren publi- \\ ziert. Die Papiere enthalten Unwahrheiten und Widersprüche, was ja auch \\ nicht erstaunlich ist, da sich die beiden Bereiche konkurrenzieren. Beide \\ Papiere sind auf www.santesuisse.ch bzw. www.consano.ch einsehbar. Der \\ Konflikt kann nur gelöst werden, wenn santésuisse sehr bald ein drittes Posi- \\ tionspapier publiziert: zum Problem der Chronischkranken, wer soll bzw. darf \\ diese betreuen.
}

\section{Cyrill Jeger}

Präsident Consano, Für eine faire und soziale Medizin in der Schweiz

1 Romanens M. Schadet das Ärzterating der medizinischen Grundversorgung? Eine Replik auf die ANOVA-Methode der santésuisse. Schweiz Ärztezeitung 2005 86(33):1911-4.

Korrespondenz:

Dr. med. Cyrill Jeger

Ziegelfeldstrasse 5

$\mathrm{CH}-4600$ Olten

jegerolten@bluewin.ch
Santésuisse hat mit der Tagung vom 23. November 2005 in Bern zur Frage der Qualität in der medizinischen Versorgung tatsächlich einen Primeur lanciert. Die Verbände der Leistungserbringer sind noch nicht soweit. Allerdings war an dieser Tagung doch die Position der santésuisseSprecher auffällig, dass Qualität nichts kosten darf - was sich auch im erwähnten neuen Positionspapier widerspiegelt. Eindrücklich an der Tagung in Bern vom 23. November war dagegen v. a. der Referent aus England, der von den grossen Anstrengungen und Investitionen des nationalen Gesundheitswesens (NHS) berichtete, um all die medizinischen Leistungserbringer in Einzel- und Gruppenpraxen elektronisch zu vernetzen, um so mit einer grosse Datenmenge Wesentliches zur Qualitätsverbesserung und Qualitätssicherung beizutragen. Eine grosse Investition, die sich für das NHS - und für die Patienten offensichtlich lohnt. Erwähnt sei aber auch, dass die Leistungserbringer erst auf staatlich verordneten Druck zu diesem Quantensprung bereit waren, und dass das NHS diesen finanzierte. Wer zusätzliche Kriterien der Qualitätssicherung erfüllt, könne mit dem NHS zu einem besseren Tarif abrechnen.

Die enormen Unterschiede struktureller Art GB gegenüber der Schweiz verhindern, dass wir hier zu ähnlichen Durchbrüchen kommen könnten, die allen Seiten - zuerst den Patientinnen und Patienten, aber auch den Leistungserbringern und den Kostenträgern - Vorteile bringen könnten. Hier wird weiter die heisse Kartoffel hin und her geschoben.

Provokativ ist folgende Behauptung von santésuisse: «Die Ärzte bzw. ihre Verbände haben bisher weder ein Qualitätskonzept noch ein Qualitätsprogramm vorgelegt.» Das grosse Netzwerk der Qualitätszirkel und die aufwendige Fortbil- dung und deren Kontrolle werden vom Tisch der santésuisse gefegt. Letztere will offensichtlich ihren politischen Druck auf die Leistungserbringer erhöhen.

Die Ernsthaftigkeit, mit der sich santésuisse neuerdings für Qualität einsetzt, muss sich noch auf dem Hintergrund der Wirtschaftlichkeitsverfahren bewähren, mit denen die gleiche santésuisse systematisch einen enormen Qualitätsabbau betreibt. Das zeigt sich auch wieder im gleichentags publizierten Positionspapier. Hier beisst sich santésuisse fest an der neu entwickelten Anova-Methode. Dabei wird auch im Papier vom Januar die ausführliche und fundierte Kritik von Dr. med. Michel Romanens an der Anova-Methode [1] nicht berücksichtigt.

Im Kern geht es eigentlich darum, dass Anova als «Feinmassage des Mittelwerts» karikiert werden kann. Dabei ist es doch hinlänglich bekannt, dass etwa $60 \%$ der Kosten einer Arztpraxis von $10 \%$ Patientinnen und Patienten benötigt bzw. verursacht werden. Dieser grundlegende «case mix» wird auch bei der «neuen» Anova nicht berücksichtigt.

Entscheidend ist nun aber, dass - abgesegnet durch höchstrichterliche Fehlentscheide - die vorgelegten Zahlen der santésuisse als Beweise einer Überarztung gelten, wo diese doch höchstens als Hinweise gelten könnten. Weltweit ist das eine einmalige Verdrehung anerkannter Rechtsgrundsätze.

Rechthaberisch beharrt santésuisse in ihrem neuen Positionspapier zur Wirtschaftlichkeit darauf, dass selbst neueste Zahlen aus den Trustzentren ihre eigenen Daten nicht in Frage stellen könnten: «Unwirtschaftliches Verhalten ist aus den Daten des santésuisse-Datenpools ${ }^{\circledR}$ sowie aus dem Tarifpool zweifelsfrei nachweisbar. Die TrustCenter können die Resultate von 
santésuisse allenfalls als Zweitmeinung bestätigen, nicht aber widerlegen.» Es gilt immerhin zu bedenken, dass unser höchstes Gericht noch nicht zur Wertung weiterer Datenquellen gesprochen hat. Zudem gilt es zu beachten, dass momentan im Parlament neue Elemente des Risikoausgleichs beraten werden. Solche neuen Kriterien müssten dann selbstredend auch bei den Wirtschaftlichkeitsverfahren berücksichtigt werden.

Es genügt auch nicht, wenn santésuisse in ihrem Positionspapier darauf hinweist, dass ihre Anova-Methode von der ETH als wissenschaftlich geprüft worden ist. Die Mittelwertberechnung wird wahrscheinlich verbessert («Feinmassage»), aber das genügt nicht, um existenzzerstörerische Rückforderungen an redliche Leistungserbringer zu richten. Consano hat auch eine wissenschaftliche Analyse der Mittelwertberechnungen in Auftrag gegeben (Matthias Schwenkglenks, Prof. Thomas D. Szucs 9. August 2005 European Center of Pharmaceutical Medicine Universität Basel www.consano.ch).

Das Resultat ist eindeutig, so eine Methode widerspricht allen anerkannten statistischen Grundsätzen. «Die Aussage der santésuisse, die Korrektur der verursachten Kosten auf Basis der Variablen Spezialisierung des Arztes, Region sowie Alter und Geschlecht der Patienten sei ausreichend, steht in scharfem Kontrast zur durchweg in der Literatur festgestellten Notwendigkeit eines casemix adjustments, das der unterschiedlichen Morbidität verschiedener Patientenkollektive adäquat Rechnung trägt.»

Dass neuerdings santésuisse nach der AnovaMethode «auffällige» Leistungserbringer zu einem Gespräch zur Darlegung der «Praxisbesonderheiten» einlädt, ist an sich schon ein Fortschritt. Nur bleiben dort die sogenannt auffälligen Leistungserbringer der reinen Willkür der regionalen santésuisse-Vertreter ausgeliefert. Unerträglich ist, dass rein wirtschaftliche Kriterien verwendet werden, um die Qualität der Leistung zu beurteilen. Das ist zudem auch gesetzwidrig, schreibt doch der berühmte Artikel 32 KVG vor, dass neben Wirtschaftlichkeit, auch die Zweckmässigkeit und die Wirksamkeit berücksichtigt werden müssen. Dass die ausführenden Bestimmungen zu diesem Artikel immer noch blankes Papier sind, stellt ein schweres Versäumnis der beteiligten Vertragspartner dar.

Qualität hat bei der aktuell geltenden Wirtschaftlichkeitsbeurteilung keinen Platz. Viele Leistungserbringer sind gezwungen, chronische und damit kostenintensivere Patienten weg- und weiterzuweisen. Das gilt besonders für Leistungserbringer, die sich (sogar nachweislich) für spezielle Patientengruppen spezialisiert haben. Da steht der Satz im anderen Positionspapier der santésuisse: «Eine Über-, Unter- oder gar Fehlversorgung ist zu vermeiden» in krassem Gegensatz - was gilt denn jetzt? Da viele Leistungserbringer gezwungen sind, kostenintensive Patienten weg- und weiterzuweisen, werden sich die Kosten nicht nur verschieben, sondern vervielfachen. Die Grundversorgung bietet gerade bei kostenintensiven Patienten eine effiziente Betreuung dar - im kleinräumigen «Kässelidenken»-, aber erst recht in der Betrachtung grösserer Zusammenhänge mit anderen Kostenträgern usw.

Consano bereitet momentan zu dieser Problematik «Chronische Patienten aus Kostengründen unterversorgt» am 27. April in Olten das zweite Symposium vor. «Die heute geltende Beurteilung der Wirtschaftlichkeit des ärztlichen Handelns (Ärzterating) schliesst den Patientenmix aus. Obwohl 10\% der Patienten einer Arztpraxis rund $60 \%$ der Kosten verursachen bzw. benötigen. Die korrekte Betreuung chronischer Patientinnen und Patienten wird für viele Ärztinnen und Ärzte existenzgefährdend, da exorbitante Rückforderungen drohen. Chronische Patienten werden wegen der notwendigen Kosten bei der Betreuung und bei den Versicherungen unbeliebter und rausgedrängt. Es folgt ein Qualitätszerfall bei Chronischkranken.»

Eine wichtige Aufgabe käme - auch nach den gesetzlichen Vorgaben(!) - dem BAG zu. Leider lässt die Verwaltung jegliche Unabhängigkeit vermissen und hat kürzlich sogar verlauten lassen, dass santésuisse gar nicht ihrer Kontrolle unterstehe. Es braucht aber eine «neutralere» Aufsicht, es kann doch nicht sein, dass der Kostenträger selber die Kosten kontrolliert. Santésuisse schreibt im Papier zu den Wirtschaftlichkeitsverfahren selber: «Zudem stellt sich die Frage, ob die Daten für eine Kontrolle von jenen stammen sollen, die kontrolliert werden.» In diesem Zitat sind scheinbar die anderen gemeint, aber die Aussage trifft auf die santésuisse genauso $\mathrm{zu}$.

Es scheint somit wirklich dringend, dass santésuisse bald ein drittes Positionspapier publiziert, das auf die Problematik der chronischen Patienten eingeht. Wer soll bzw. darf diese betreuen, wie haben sich die Leistungserbringer abzusichern usw.?

Sollen die komplexen Probleme im Gesundheitswesen - es geht immer um die Patientinnen und Patienten - effektiv gelöst werden, braucht es etwas mehr Kooperation. Um die aktuelle Konfrontation zu überwinden, sind Schritte von allen Seiten notwendig. 